\title{
Relação da ractopamina com componentes nutricionais e desempenho em suínos: um estudo meta-analítico
}

\author{
Relationship among ractopamine, nutritional variables and performance in pigs: a meta-analytic study
}

\author{
Ines Andretta ${ }^{I^{*}, \text { III }}$ Paulo Alberto Lovatto ${ }^{I}$ Marcos Kipper da Silva ${ }^{\mathrm{I}, \text { II }}$ Cheila Roberta Lehnen ${ }^{\mathrm{I}, \text { III }}$ \\ Eloiza Lanferdini, ${ }^{\mathrm{I}, \mathrm{III}}$ Cristieli Carolina Klein ${ }^{\mathrm{I}, \mathrm{IV}}$
}

RESUMO

Uma meta-análise foi realizada para avaliar a associação da ractopamina com variáveis nutricionais e de desempenho em suinos. A base de dados contemplou 29 artigos publicados em periódicos nacionais e internacionais entre 1990 e 2007, totalizando 155 tratamentos e 3.786 suinos em terminação. A inclusão média de ractopamina foi de 15,3ppm (variação de 0 a 30ppm) e a ingestão média diária de ractopamina foi 24,9mg (variação de 0 a $84 \mathrm{mg}$ ). A inclusão de ractopamina nas dietas apresentou $(P<0,05)$ correlação positiva com o ganho de peso $(0,214)$ e com a eficiência alimentar dos animais $(0,231)$. A ractopamina não alterou $(P>0,05)$ o consumo de ração, mas aumentou $(P<0,05) \mathrm{em}$ cerca de $12 \%$ a eficiência alimentar, em $10 \%$ o ganho de peso e em 3,7\% o peso vivo final dos animais. As concentrações de proteina, lisina e energia nas dietas afetaram a resposta dos animais suplementados com ractopamina. Para cada incremento em uma unidade percentual no conteúdo dietético de lisina, pode ser esperado aumento de $341 \mathrm{~g}$ no ganho de peso $\left(G M D=0,687+0,341 L I S ; R^{2}=0,71\right)$ e de $1,3 \%$ na eficiência alimentar dos animais $\left(E F=2,14+1,34 L I S ; R^{2}=0,62\right)$. A ractopamina melhora o desempenho dos suinos, porém a interação do aditivo com os componentes nutricionais deve ser considerada para a formulação das dietas.

Palavras-chave: agonistas, lisina, metabolismo, $\beta$-adrenérgicos.

\section{ABSTRACT}

A meta-analysis was carried to evaluate the relationship of ractopamine with nutritional variables and performance in pigs. The database included 29 articles published in national and international journals from 1990 to 2007, totaling 155 treatments and 3,786 pigs. The average inclusion of ractopamine was $15.3 \mathrm{ppm}$ (ranged from 0 to $30 \mathrm{ppm}$ ) and the daily average intake of ractopamine was $24.9 \mathrm{mg}$ (ranging from 0 to $84 \mathrm{mg}$ ). Inclusion of ractopamine in diets showed $(P<0.05)$ a positive correlation with weight gain (0.214) and feed efficiency (0.231). Ractopamine did not change $(P>0.05)$ the feed intake, but increased $(P<0.05)$ in $12 \%$ the feed efficiency, in $10 \%$ the weight gain and in $3.7 \%$ the final body weight. The concentrations of protein, lysine and energy in diets affected the animal response feeding with ractopamine. Increasing a unit in dietary lysine concentration resulted in increase of $341 \mathrm{~g}$ in weight gain $\left(A D G=0.687+0.341 L I S, R^{2}=0.71\right)$ and $1.3 \%$ in feeding efficiency $\left(E F=2.14+1.34 L I S ; \quad R^{2}=0.62\right)$. Ractopamine improves pig performance, however, the interaction between additive and nutritional components must be considered in diets formulation.

Key words: agonists, lysine, metabolism, $\beta$-adrenergics.

\section{INTRODUÇÃO}

A ractopamina, agonista $\beta$-adrenérgico do grupo das fenetanolaminas, é uma substância exógena que altera a partição dos nutrientes para deposição muscular e de gordura. Trata-se de um análogo sintético oralmente ativo das catecolaminas, hormônios que regulam vários processos fisiológicos pela ativação

IGrupo de Modelagem Animal (Gmodel/CNPq), Departamento de Zootecnia, Universidade Federal de Santa Maria (UFSM), 97105900, Santa Maria, RS, Brasil. E-mail: iandretta@gmail.com. *Autor para correspondência.

"Programa de Pós-graduação em Medicina Veterinária, UFSM, Santa Maria, RS, Brasil.

IIIPrograma de Pós-graduação em Zootecnia, UFSM, Santa Maria, RS, Brasil.

${ }^{\mathrm{IV}}$ Curso de Zootecnia, UFSM, Santa Maria, RS, Brasil. 
de receptores adrenérgicos específicos (RAMOS \& SILVEIRA, 2001). Em nível celular, a ractopamina pode inibir a lipogênese e estimular a lipólise (MILLS, 2002). Além disso, a atividade do agonista está relacionada com a modulação de diversas enzimas envolvidas no metabolismo lipídico e glicídico (RAMOS \& SILVEIRA, 2001).

Os aditivos $\beta$-adrenérgicos são uma alternativa nutricional comumente utilizada na produção animal como particionadores de nutrientes, melhorando a retenção de nitrogênio, as taxas de crescimento e a deposição de tecido muscular em detrimento de gordura (ARMSTRONG et al., 2004). Apesar do amplo uso em nutrição animal, pouco é conhecido sobre as implicações da ractopamina como aditivo melhorador de desempenho, especialmente no que diz respeito à sua interação com outros componentes nutricionais.

As alterações metabólicas causadas pela ractopamina podem interferir nas exigências nutricionais dos animais. Assim, um aumento substancial no ganho de peso, por exemplo, estaria relacionado com a necessidade de um maior aporte de lisina nas dietas, visto a relação direta entre esse nutriente e a deposição proteica (APPLE et al., 2004). Apesar disso, o aditivo muitas vezes é utilizado a campo sem que a base nutricional das dietas seja alterada. Essa condição ignora as possíveis alterações nas exigências dos suínos e representa um prejuízo produtivo, à medida que não permite aos animais a expressão de seu potencial máximo de desempenho.

Nesse contexto, o uso da meta-análise como ferramenta capaz de gerar novos resultados, poderia integrar diferentes variáveis de artigos anteriormente publicados e estabelecer respostas sistêmicas e ajustadas à diversidade experimental. Este trabalho foi realizado, portanto, com o objetivo de estudar, através de uma meta-análise, a relação do fornecimento de ractopamina com componentes nutricionais e variáveis de desempenho em suínos.

\section{MATERIAL E MÉTODOS}

O trabalho foi realizado no Grupo de Modelagem Animal (GModel/CNPq) do Departamento de Zootecnia da Universidade Federal de Santa Maria. A base de dados utilizada para a meta-análise foi composta por 29 artigos (disponíveis para consulta com os autores). A base espacial foi a de periódicos nacionais e internacionais (Pesquisa Agropecuária Brasileira, Revista Brasileira de Zootecnia, Journal of Animal Science, Animal Feed Science and Technology e Meat Science), e a base temporal compreendeu publicações entre 1990 e 2007. Das publicações utilizadas na base, 20 tiveram origem em institutos americanos. Os demais trabalhos foram realizados no Brasil (4), Austrália (2), Canadá (2) e China (1).

Os principais critérios de seleção das publicações foram (a) adição de ractopamina nas dietas, (b) genéticas (raças e/ou cruzamentos industriais), (c) fase alimentar (terminação) e (d) resultados que incluíam avaliações de desempenho. A metodologia para a definição das variáveis dependentes e independentes e para a codificação dos dados seguiu as proposições descritas na literatura (LOVATTO et al., 2007; SAUVANT et al., 2008).

As informações foram selecionadas nas seções do material e métodos e dos resultados de cada artigo e tabuladas em uma base elaborada em planilha de dados. As variáveis analisadas foram relativas às características experimentais (período experimental e de fornecimento das dietas suplementadas, inclusão da ractopamina nas dietas, idade, peso vivo, sexo e lotação), à composição nutricional das dietas, ao consumo de nutrientes (proteína bruta, lisina total e energia metabolizável) e ao desempenho (consumo de ração, ganho de peso e eficiência alimentar). A eficiência alimentar calculada foi obtida pela relação entre os valores apresentados de consumo de ração e ganho de peso.

A base foi composta de 155 dietas e 3.786 animais (fêmeas e machos castrados). $\mathrm{O}$ peso médio dos animais ao início dos experimentos foi de $71,3 \mathrm{~kg}$ e de $103,3 \mathrm{~kg}$ ao abate. O milho e o farelo de soja foram as principais fontes energética e proteica utilizadas, em 55 e $64 \%$ das dietas, respectivamente. A densidade nutricional média das dietas foi de $3.381 \mathrm{kcal}$ de energia metabolizável (mínimo: 2.960kcal; máximo: 3.770kcal; coeficiente de variação: $4 \%$ ) e de $17,6 \%$ de proteína bruta (mínimo: 8,4\%; máximo: 24\%; coeficiente de variação: $16 \%$ ). $O$ teor médio de lisina total nas dietas foi de $1,04 \%$, com mediana em $1,12 \%$ e coeficiente de variação de $12,4 \%$. Para fins de comparação, foram calculados os consumos de lisina por unidade (quilograma) de peso metabólico $\left(\mathrm{PV}^{0,6}\right)$ e classificados como níveis acima e abaixo da média geral (195mg de lisina $\left.\mathrm{kg}^{-1} \mathrm{PV}^{0,6}\right)$.

A concentração média de ractopamina nas dietas foi de $15,3 \mathrm{ppm}$, variando de 0 a 30 ppm na ração. Considerando o consumo de ração, a ingestão de ractopamina variou de 0 a $84 \mathrm{mg}$, sendo a ingestão média de $24,9 \mathrm{mg}$ por dia. O período médio de fornecimento das rações experimentais foi de 38 dias (variação de 6 a 88). O número de animais por baia foi em média de 3 (variando de 1 a 13), sendo utilizado espaçamento médio de $2,5 \mathrm{~m}^{2}$ por animal. A temperatura média nas instalações foi de $20,3^{\circ} \mathrm{C}$ (variação de 18,3 a 25,5). 
A meta-análise foi realizada segundo LOVATTO etal. (2007) e SAUVANT et al. (2008) e seguiu três análises sequenciais: gráfica (para observar a coerência biológica dos dados), de correlação (entre as diversas variáveis, para identificar os fatores relacionados na base) e de variância. Foram utilizados, nos modelos para as análises de variância e covariância, os fatores com coeficientes de correlação mais elevados e as codificações para os efeitos inter e intra-experimentos (LOVATTO et al., 2007). As equações de regressão foram obtidas através da análise de variância-covariância através do procedimento GLM. Todas as análises foram realizadas através do programa MINITAB 15 (2007).

\section{RESULTADOS E DISCUSSÃO}

Suínos alimentados com dietas contendo ractopamina apresentaram consumo de ração semelhante $(\mathrm{P}>0,05)$ aos que receberam dietas não suplementadas (Tabela 1). A inclusão de ractopamina nas dietas apresentou correlação positiva com o ganho de peso e com a eficiência alimentar dos animais. Os coeficientes de correlação relativamente baixos podem indicar que outros aspectos, além da inclusão de ractopamina nas dietas, atuaram nos índices de desempenho.

A ractopamina aumentou $(\mathrm{P}<0,05) \mathrm{em} 10 \%$ o ganho de peso e em $3,7 \%$ o peso vivo dos animais ao final dos experimentos. Segundo as equações obtidas por análise de covariância contínua (Tabela 2), para cada aumento de $1 \mathrm{mg}$ de ractopamina por quilograma de dieta, pode ser esperado incremento de $0,008 \mathrm{~g}$ no ganho de peso diário dos suínos. Apesar disso, a resposta dos animais não se apresenta constante durante o tempo, sendo maior no início da suplementação e reduzindo com o passar do período de fornecimento.

As alterações no desempenho dos animais suplementados com ractopamina podem estar associadas com respostas celulares, que incluem estímulo da lipólise, neoglicogênese e glicogenólise (MILLS, 2002). Assim, atuando no metabolismo dos animais como um particionador de nutrientes para funções desejáveis, a ractopamina pode melhorar as taxas de crescimento e a eficiência alimentar de suínos (ARMSTRONG et al., 2004).

Os resultados obtidos através da metaanálise indicam que a ractopamina aumentou $(\mathrm{P}<0,05)$ em $14 \%$ a eficiência alimentar observada e em $11 \%$ a eficiência alimentar calculada dos animais. Para cada aumento de $1 \mathrm{mg}$ de ractopamina por quilograma de dieta, pode ser esperado um incremento de $0,002 \%$ na eficiência alimentar observada.

Diversos trabalhos relatam melhorias na eficiência alimentar dos suínos quando suplementados com ractopamina, apontando esse parâmetro como um dos mais beneficiados pelo aditivo (RAMOS \& SILVEIRA, 2002; PEREIRA et al., 2008). O direcionamento de nutrientes para a deposição de tecido magro é uma das justificativas para a melhora na eficiência alimentar, uma vez que o envolvimento de

Tabela 1 - Desempenho, obtido por meta-análise, de suínos em função da ractopamina nas dietas.

\begin{tabular}{|c|c|c|c|}
\hline Variáveis & \multicolumn{2}{|c|}{ Médias } & Correlação com a inclusão de ractopamina na dieta \\
\hline \multicolumn{4}{|l|}{ Consumo diário de ração $(\mathrm{kg})$} \\
\hline Controle & $2,751^{\text {ns }}$ & \multirow{2}{*}{$\mathrm{dpr}=0,108$} & \multirow{2}{*}{$0,017^{\text {ns }}$} \\
\hline Dietas com ractopamina & $2,735^{\mathrm{ns}}$ & & \\
\hline \multicolumn{4}{|l|}{ Ganho diário de peso $(\mathrm{kg})$} \\
\hline Controle & $0,845 * *$ & \multirow{2}{*}{$\mathrm{dpr}=0,078$} & \multirow{2}{*}{$0,214^{* *}$} \\
\hline Dietas com ractopamina & $0,931 * *$ & & \\
\hline \multicolumn{4}{|c|}{ Eficiência alimentar observada } \\
\hline Controle & $0,312 *$ & \multirow{2}{*}{$\mathrm{dpr}=0,039$} & \multirow{2}{*}{$0,231 *$} \\
\hline Dietas com ractopamina & $0,356 *$ & & \\
\hline \multicolumn{4}{|c|}{ Eficiência alimentar calculada } \\
\hline Controle & $0,305 *$ & \multirow{2}{*}{$\mathrm{dpr}=0,039$} & \multirow{2}{*}{$0,153 *$} \\
\hline Dietas com ractopamina & $0,339 *$ & & \\
\hline \multicolumn{4}{|l|}{ Peso vivo final $(\mathrm{kg})$} \\
\hline Controle & $99,14 * *$ & \multirow[b]{2}{*}{$\mathrm{dpr}=6,12$} & \multirow[b]{2}{*}{$0,331^{\mathrm{ns}}$} \\
\hline Dietas com ractopamina & $102,82 * *$ & & \\
\hline
\end{tabular}

dpr Desvio padrão residual.

Probabilidade: ns (não-significativo); * $\mathrm{e}^{* *}$ (significativo a 5 e $1 \%$ de probabilidade, respectivamente, pelo teste $\mathrm{F}$ ). 
Tabela 2 - Equações para variáveis de desempenho obtidas por análise de variância-covariância.

\begin{tabular}{|c|c|c|c|c|}
\hline Respostas & Covariáveis & dpr & Equações & $\mathrm{R}^{2}$ \\
\hline \multirow{5}{*}{ Consumo diário de ração $(\mathrm{kg})$} & $\mathrm{R}$ & 0,289 & $2,938-0,010 \mathrm{R}$ & 0,59 \\
\hline & $\mathrm{R}, \mathrm{D}$ & 0,285 & $3,074-0,008 \mathrm{R}-0,007 \mathrm{D}$ & 0,60 \\
\hline & $\mathrm{P}, \mathrm{P}^{2}, \mathrm{R}, \mathrm{D}$ & 0,168 & $-11,96+0,2982 \mathrm{P}-0,0015 \mathrm{P}^{2}-0,0061 \mathrm{R}-0,0074 \mathrm{D}$ & 0,76 \\
\hline & $\mathrm{R}, \mathrm{PB}$ & 0,290 & $2,828-0,010 \mathrm{R}+0,006 \mathrm{~PB}$ & 0,69 \\
\hline & R, LIS & 0,306 & $3,028-0,010 \mathrm{R}-0,052 \mathrm{LIS}$ & 0,43 \\
\hline \multirow{5}{*}{ Ganho diário de peso $(\mathrm{kg})$} & $\mathrm{R}$ & 0,151 & $0,999+0,008 \mathrm{R}$ & 0,53 \\
\hline & $\mathrm{R}, \mathrm{D}$ & 0,141 & $1,142+0,006 \mathrm{R}-0,005 \mathrm{D}$ & 0,60 \\
\hline & $\mathrm{P}, \mathrm{P}^{2}, \mathrm{R}, \mathrm{D}$ & 0,073 & $-6,263+0,1597 \mathrm{P}-0,0008 \mathrm{P}^{2}+0,0033 \mathrm{R}-0,0085 \mathrm{D}$ & 0,82 \\
\hline & $\mathrm{R}, \mathrm{PB}$ & 0,139 & $0,547+0,008 \mathrm{R}+0,025 \mathrm{~PB}$ & 0,71 \\
\hline & R, LIS & 0,024 & $0,719+0,005 \mathrm{R}+0,286 \mathrm{LIS}$ & 0,48 \\
\hline \multirow{5}{*}{ Eficiência alimentar } & $\mathrm{R}$ & 0,038 & $0,346+0,002 \mathrm{R}$ & 0,55 \\
\hline & $\mathrm{R}, \mathrm{D}$ & 0,033 & $0,421+0,001 R-0,002 \mathrm{D}$ & 0,66 \\
\hline & $\mathrm{P}, \mathrm{P}^{2}, \mathrm{R}, \mathrm{D}$ & 0,073 & $-0,519+0,0222 \mathrm{P}-0,0001 \mathrm{P}^{2}+0,0004 \mathrm{R}-0,0022 \mathrm{D}$ & 0,80 \\
\hline & $\mathrm{R}, \mathrm{PB}$ & 0,038 & $0,142+0,001 \mathrm{R}+0,011 \mathrm{~PB}$ & 0,68 \\
\hline & R, LIS & 0,028 & $0,396+0,002 \mathrm{R}-0,031 \mathrm{LIS}$ & 0,64 \\
\hline
\end{tabular}

${ }^{\text {dpr }}$ Desvio padrão residual.

${ }^{\mathrm{R}}$ Inclusão de ractopamina na dieta (ppm).

${ }^{\mathrm{P}}$ Peso vivo (kg).

${ }^{\mathrm{PB}}$ Proteína bruta na dieta (\%).

LIS Lisina na dieta (\%).

${ }^{\mathrm{D}}$ Período de fornecimento das dietas experimentais (dias).

grande quantidade de água na síntese de tecido muscular representa, metabolicamente, uma economia energética para o animal, quando comparada com a deposição de tecido adiposo.

Considerando apenas os animais suplementados com ractopamina, o teor de lisina total das dietas apresentou correlação positiva com o ganho de peso e com a eficiência alimentar calculada. Nos animais que recebiam dietas com ractopamina, o ganho de peso e a eficiência alimentar aumentaram $(\mathrm{P}<0,05)$ com o consumo de lisina (Tabela 3). Para cada incremento em uma unidade percentual no conteúdo dietético de lisina, pode ser esperado aumento de $341 \mathrm{~g}$ no ganho de peso (GMD $=0,687+0,341$ LIS; $\left.\mathrm{R}^{2}=0,71\right) \mathrm{e}$ de $1,3 \%$ na eficiência alimentar dos animais $\left(E F=2,14+1,34\right.$ LIS; $\left.R^{2}=0,62\right)$. Além disso, os suínos suplementados com ractopamina e com consumo diário de lisina superior à média (mais de $195 \mathrm{mg} \mathrm{kg}^{-1} \mathrm{PV}^{0,6}$ ) apresentaram ganho de peso $20 \%$ maior $(\mathrm{P}<0,05)$ e eficiência alimentar $5 \%$ melhor em relação aos animais que recebiam menores quantidades do aminoácido.

O conteúdo de lisina nas dietas afeta a magnitude com que os suínos respondem a ractopamina (APPLE et al., 2004). Nos animais suplementados, o aumento na densidade de lisina nas dietas pode ser associado com a redução no consumo voluntário de alimento e com o incremento no ganho de peso e na eficiência alimentar (DUNSHEA et al., 1993; WEBSTER et al., 2007). Essa interferência pode ser relacionada com a ação dos $\beta$-adrenérgicos sobre o ganho de peso e o perfil de aminoácidos depositados na massa muscular (REEDS \& MERSMANN, 1991). A concentração de lisina na proteína depositada por suínos suplementados com ractopamina aumenta em cerca de 5\% (SCHINCKEL et al., 2003). Assim, é provável que as ações mediadas pela ractopamina alterem as exigências do aminoácido, o que determinaria ajustes nutricionais nas dietas suplementadas (MITCHELL et al., 1990; MITCHELL et al., 1991).

A lisina pode alterar as respostas produtivas nos suínos que recebem dietas com ractopamina, especialmente no início da suplementação, quando as respostas ao aditivo são ainda mais evidentes. Além do período, a dose de ractopamina utilizada pode interferir nas respostas dos suínos aos níveis de lisina (WEBSTER et al., 2007).

Outros componentes nutricionais são apontados como moduladores no desempenho dos suínos alimentados com dietas contendo ractopamina. A figura 1 mostra que, em animais suplementados, o ganho de peso aumenta em função do consumo de proteína bruta e da energia metabolizável.

Alguns estudos sugerem que o nível de proteína altera a eficiência da ractopamina em promover a retenção de nitrogênio, sendo esta mais efetiva quando o consumo proteico é maior (REEDS \& 
Tabela 3 - Ganho de peso e eficiência alimentar, obtidos por meta-análise, de suínos alimentados com ractopamina em função do consumo diário de lisina por unidade de peso metabólico.

\begin{tabular}{|c|c|c|c|c|}
\hline & \multicolumn{2}{|c|}{----Consumo diário de lisina $\mathrm{kg}^{-1} \mathrm{PV}^{0,6}$} & \multirow{2}{*}{ dpr } & \multirow{2}{*}{$\begin{array}{c}\text { Correlação com o teor de lisina } \\
\text { total nas dietas }\end{array}$} \\
\hline & Abaixo da média ${ }^{1}$ & Acima da média & & \\
\hline Ganho diário de peso $(\mathrm{kg})$ & $0,885^{* * *}$ & $1,060^{* * *}$ & 0,015 & $0,262^{*}$ \\
\hline Efic. alimentar observada & $0,364^{*}$ & $0,358^{*}$ & 0,018 & $0,554^{* * *}$ \\
\hline Efic. alimentar calculada & $0,352^{*}$ & $0,355^{*}$ & 0,011 & $0,513^{* * *}$ \\
\hline
\end{tabular}

${ }^{1}$ Consumo médio de $195 \mathrm{mg}$ de lisina $\mathrm{kg}^{-1} \mathrm{PV}^{0,6}$.

${ }^{\text {dpr }}$ Desvio padrão residual.

Probabilidade: $* * * \mathrm{e}^{* * *}$ (significativo a 5,1 e $0,1 \%$ de probabilidade pelo teste $\mathrm{F}$ ).

MERSMANN, 1991). Além do conteúdo de proteína, parte do efeito da ractopamina pode ser associada com a densidade energética das dietas, como descrito por MITCHELL et al. (1991), que observaram uma relação linear entre a ingestão de energia e a retenção total de energia, de proteína e de lipídios.

A relação da energia dietética com o desempenho dos suínos alimentados com dietas contendo ractopamina ainda é controversa, uma vez que outros autores não observaram efeito da densidade energética sobre o ganho de peso dos suínos suplementados (APPLE et al., 2004). Por outro lado, a relação lisina:energia nas dietas parece afetar linearmente o ganho de peso e a eficiência alimentar dos animais (CASTELL et al., 1994; APPLE et al., 2004). É provável que o uso de aditivos adrenérgicos esteja relacionado com um aumento nas exigências de energia de mantença (REEDS \& MERSMANN, 1991), mas que se relacione mais estritamente com as exigências de proteína e aminoácidos dos animais.

Muitos trabalhos vêm sendo conduzidos para avaliar os efeitos da ractopamina nos índices produtivos de suínos. A associação da ractopamina com as alterações metabólicas também tem sido objeto de diversas pesquisas. Porém, os efeitos do aditivo ainda não estão bem reportados. A meta-análise permitiu estudar o tema de forma sistemática e os resultados obtidos pela nova abordagem mostram que

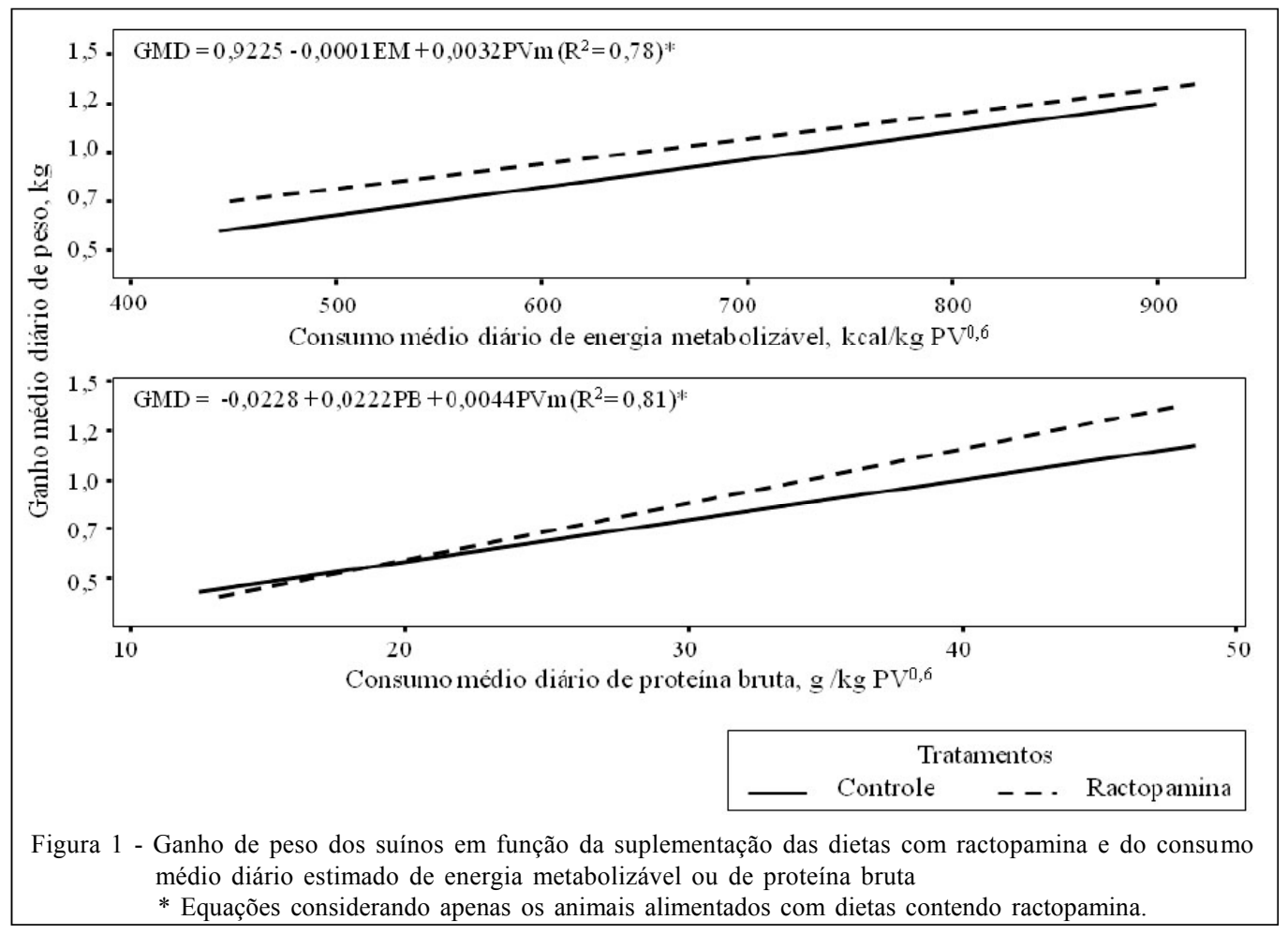

Ciência Rural, v.41, n.1, jan, 2011. 
a ractopamina não altera o consumo de ração, mas melhora o ganho de peso e a eficiência alimentar dos suínos. Esses resultados também evidenciaram que a combinação apropriada entre o aditivo e os diversos componentes nutricionais devem ser considerados, indicando a necessidade de novas pesquisas com esse objetivo.

\section{CONCLUSÃO}

A ractopamina não altera o consumo de ração, mas melhora o ganho de peso e a eficiência alimentar dos suínos. Animais suplementados com ractopamina e alimentados com dietas contendo níveis mais altos de lisina total apresentam maior ganho de peso e melhor eficiência alimentar.

\section{REFERÊNCIAS}

APPLE, J.K. et al. Effects of dietary lysine and energy density on performance and carcass characteristics of finishing pigs fed ractopamine. Journal of Animal Science, v.82, p.32773287, 2004. Disponível em: <http://jas.fass.org/cgi/content/ full/82/11/3277>. Acesso em: 20 dez. 2009.

ARMSTRONG, T.A. et al. The effect of dietary ractopamine concentration and duration of feeding on growth performance, carcass characteristics, and meat quality of finishing pigs. Journal of Animal Science, v.82, p.3245-3253, 2004. Disponível em: <http://jas.fass.org/cgi/content/full/82/11/ 3245>. Acesso em: 16 dez. 2009.

CASTELl, A.G. et al. Performance, carcass and pork characteristics of castrates and gilts self-fed diets differing in protein content and lysine:energy ratio. Canadian Journal of Animal Science, v.74, p.519-528, 1994.

DUNSHEA, F.R. et al. Interrelationships between dietary protein and ractopamine on protein and lipid deposition in finishing gilts. Journal of Animal Science, v.71, p.29312941, 1993. Disponível em: <http://jas.fass.org/cgi/content/ full/82/11/2931>. Acesso em: 13 dez. 2009.

LOVATTO, P.A. et al. Meta-análise em pesquisas científicas enfoque em metodologias. Revista Brasileira de Zootecnia, v.36, p.285-294, 2007.

MILLS, S.E. Biological basis of the ractopamine response. Journal of Animal Science, v.80 (Suppl. 2), p.E28-E32, 2002. Disponível em: <http://jas.fass.org/cgi/reprint/80/ESuppl_2/E28>. Acesso em: $10 \mathrm{dez} .2009$.
MINITAB. User's guide: Meet Minitab 15, Stat College, PA, 2007. 142p.

MITCHELL, A.D. et al. Response of low and high protein select lines of pigs to the feeding of the beta-adrenergic agonist ractopamine (phenethanolamine). Journal of Animal Science, v.68, p.3226-3232, 1990. Disponível em: <http:// jas.fass.org/cgi/reprint/68/10/3226>. Acesso em: Acesso em: 21 dez. 2009

MITCHELL, A.D. et al. Influence of level of dietary protein or energy on effects of ractopamine in finishing swine. Journal of Animal Science, v.69, p.4487-4495, 1991. Disponível em: <http://jas.fass.org/cgi/reprint/69/11/4487>. Acesso em: 20 dez. 2009.

PEREIRA, F.A. et al. Efeitos da ractopamina e de dois níveis de lisina digestível na dieta sobre o desempenho e características de carcaça de leitoas em terminação. Arquivo Brasileiro de Medicina Veterinária e Zootecnia, v.60, p.943-952, 2008.

RAMOS, F.; SILVEIRA, M.I.N.D. Agonistas adrenérgicos $\beta 2$ e produção animal: II - Relação estrutura-actividade e farmacocinética. Revista Portuguesa de Ciências Veterinárias, v.96, p.167-175, 2001. Disponível em: <http:/ /www.fmv.utl.pt/spcv/PDF/pdf12_2001/Agonistas\%20.pdf>. Acesso em: 20 dez. 2009.

RAMOS, F.; SILVEIRA, M.I.N.D. Agonistas adrenérgicos B2 e produção animal: III - Efeitos zootécnicos e qualidade da carne. Revista Portuguesa de Ciências Veterinárias, v.97, p.5162, 2002. Disponível em: <http://www.fmv.utl.pt/spcv/PDF/ pdf6_2002/RPCV542_51-62.PDF>. Acesso em: 22 dez. 2009.

REEDS, P.J.; MERSMANN, H.J. Protein and energy requirements of animals treated with beta-adrenergic agonists: a discussion. Journal of Animal Science, v.69, p.15321550, 1991. Disponível em: $<\mathrm{http}: / /$ jas.fass.org/cgi/reprint/ 69/4/1532>. Acesso em: 14 dez. 2009.

SAUVANT, D. et al. Meta-analyses of experimental data in animal nutrition. Animal, v.2, p.1203-1214, 2008. Acesso em: 10 set. 2009

SCHINCKEL, A.P. et al. Development of a model to describe the compositional growth and dietary lysine requirements of pigs fed ractopamine. Journal of Animal Science, v.81, p.1106-1119, 2003. Disponível em: <http://jas.fass.org/cgi/ reprint/81/5/1106>. Acesso em: 13 dez. 2009.

WEBSTER, M.J. et al. Interactive effects between ractopamine $\mathrm{HCl}$ and dietary lysine on finishing pig growth performance, carcass characteristics, pork quality and tissue accretion. Professional Animal Scientist, v.23, p.597-611, 2007. Disponível em: <http://pas.fass.org/content/23/6/ 597.full.pdf + html>. Acesso em: 20 dez. 2009. 